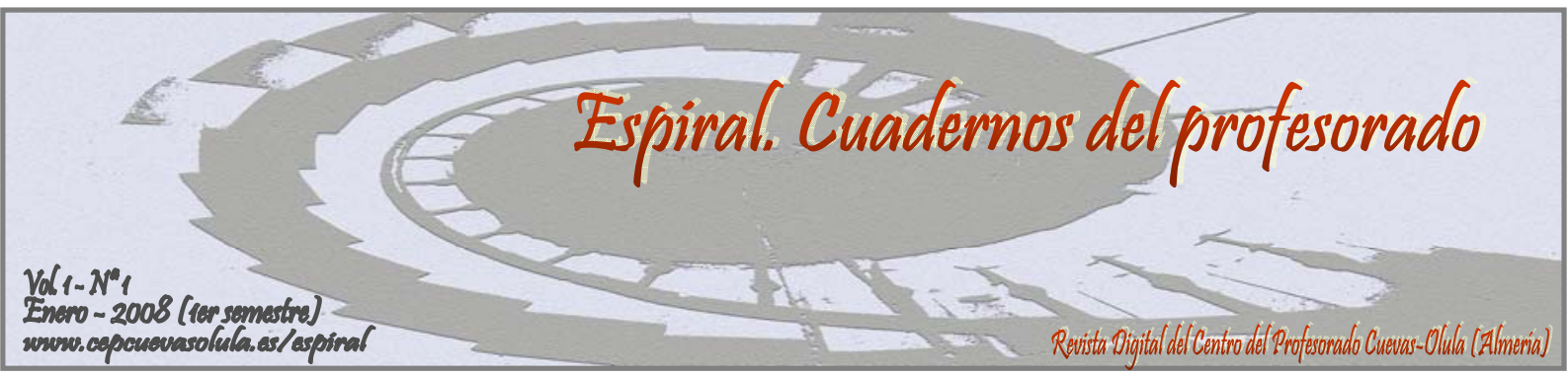

\title{
ELABORACIÓN Y CONSTRUCCIÓN DE MATERIALES PARA EL BLOQUE DE CONTENIDOS DE ACTIVIDAD FÍSICA EN EL MEDIO NATURAL: EL ROCÓDROMO DE ESCALADA.
}

\author{
Antonio Baena Extremera y Juan Francisco Calvo Morales
}

\author{
I.E.S. Cardenal Cisneros, Albox (Almería)
}

Enviar correspondencia a: abaenaextrem@yahoo.es

\begin{abstract}
RESUMEN: El presente trabajo pretende mostrar una experiencia práctica en la construcción de materiales didácticos en Educación Física. Primero se ha buscado dentro del centro el lugar más idóneo para el rocódromo, luego se ha diseñado cumpliendo unas características y una vez comprado los materiales necesarios, se llevó a cabo su construcción. Este recurso servirá para realizar varias unidades didácticas en diferentes cursos y etapas. Además, ayudará a conseguir tanto objetivos de etapa, como de área y de programación, así como contenidos propios del área como de otras materias y de temas transversales.
\end{abstract}

Palabras clave: rocódromo, escalada, actividad física en el medio natural, material didáctico.

ABSTRACT: The actual project is intended to describe a practical experience for the building of didactic materials for de Physical Education subject. First of all we found out a suitable location for the inside climbing centre in the school. Then the centre was designed according to some particular features. Finally, it was built after getting the necessary material. Such a resource will be useful for performing several didactic units in different groups and stages. It will also help to achieve some of the aims programmed as well as the contents both in the area and other cross-curricular activities.

Key words: inside climbing centre, climbing, outdoor activities, didactic material

1.- INTRODUCCIÓN: ORIGEN DE LAS ACTIVIDADES FÍSICAS EN EL MEDIO NATURAL EN LA ENSEÑANZA.

En la primera mitad del s. XX, la actividad física en el medio natural se institucionaliza, aparece un mayor abanico de actividades y algunas de ellas se diferencian llegando a deportivizarse. En España, las ideas pedagógicas de Rousseau se concretan en Institución Libre de Enseñanza (ILE), siendo uno de los fenómenos educativos y culturales más relevantes de la España contemporánea (López, 1998, p. 29). Para la Educción Física supone un evidente avance, al ser la entidad que de forma más entusiasta la introduce en el ámbito educativo (Pastor Pradillo, 1997). Las ideas de renovación pedagógica, en el fondo, son fruto de una necesaria adaptación a las condiciones de infraestructuras escolares de la época, producida por una falta de espacios verdes de las ciudades (Devís, 1994). Las Escuelas del Ave María (Manjón) responden a estas demandas, creándose centros en pleno contacto con la naturaleza, con un gran jardín, con prados, bosquecillos,... (Carreño, 2000).

Las actividades en la naturaleza son una constante en los programas de la ILE, 
desarrollándose dentro del marco escolar curricular. Por eso, eran organizadas por los propios maestros de la escuela. Asistía un maestro por cada diez alumnos. Se desarrollaban en espacios naturales diversos, que tuvieran interés paisajístico e histórico, lo que demuestra que la naturaleza es considerada como una escuela de aprendizajes. Se desarrollaban actividades de carácter físico (baños, paseos, juegos) y actividades de carácter académico: "adquirir sin esfuerzo y de manera intuitiva muchas ideas de geografía,...” (Cossío, 1888, p. 207).

En el siglo XXI, las Actividades en la naturaleza se introducen dentro de los programas educativos de Primaria y Secundaria. En primaria, a través de los bloques de contenidos de Salud, el Juego y Conocimiento y Desarrollo Corporal (Decreto 105/1992 que establece el currículum para la Educación Primaria en Andalucía) y en Secundaria a través del Bloque de Actividad Física en el Medio Natural (Decreto 106/1992 modificado por el Decreto 148/2002 que establece el currículum para la Educación Secundaria en Andalucía, y Decreto 208/2002 que modifica al Decreto 126/1994 que establece el currículum para el Bachillerato en Andalucía). Con la nueva implantación de la Ley 2/2006 de Educación, se aprueba el Real Decreto 1631/2006, de 29 de Diciembre por el que se establecen las enseñanzas mínimas en la ESO, dentro del cual se dedica el bloque 4 a las Actividades en la Naturaleza.

\section{2.- ORIGEN DE LA EXPERIENCIA.}

Desde diferentes puntos de la geografía mundial se está haciendo un llamamiento a reconsiderar los planteamientos tradicionales, que desde hace ya décadas, se llevan utilizando en los contenidos del área de Educación Física. Autores como Dewar (1991), Carlson (1995), Povenius y Romar (1995), Sage (1998), Lagardera (2000) y Fernández-Río et al. (2003), se plantean la enseñanza de nuestra área desde otra perspectiva y con la utilización de otros contenidos educativos, antes menos trabajados y día a día más incluidos en las programaciones de Educación Física.

Dentro del gran elenco de actividades posibles a desarrollar en nuestro entorno, podemos diferenciar aquellas cuyo medio no es determinante para el tipo de respuesta, es decir, que se realizan igual en el aire libre que en un recinto cerrado; y aquellas en las que el entorno sí es determinante e influyente para desarrollar una actividad concreta (como el piragüismo). Pero existe una clara diferencia entre la puesta en práctica de actividades que:

$\square$ Son desarrolladas en un gimnasio, piscina, sala o pista deportiva y que son transportadas a un entorno natural.

$\square$ Actividades que son desarrolladas en el medio natural y se llevan o intentan llevar al escenario natural de la educación física escolar.

$\square$ Y el grupo de actividades que solamente pueden ser realizadas en este entorno medioambiental.

García y Quintana (2005, p.12) creen que desde una perspectiva educativa, las actividades en la naturaleza se podrían agrupar en 5 niveles, dependiendo del marco en el que se desarrollen:

$\square$ Marco Cerrado: Se desarrollan en el interior del aula o centro, como apoyo o información de las que haremos en el campo o como actividades en sí mismas.

$\square$ Marco Abierto: Se realizan en plena naturaleza.

$\square$ Marco Mixto: Exigen parte de preparación en el centro o espacios alternativos y otra en el campo.

$\square$ Marco Alternativo cercano: Se dan en espacios próximos a los centros de enseñanza, parques, plazas, calles, etc.

$\square$ Marco Alternativo lejano: Son las realizadas en instalaciones lejanas al centro, pero acondicionadas, como son los equipamientos ambientales, campamentos, rocódromos, etc.

Estas prácticas, consideradas hoy en día de innovación educativa, tendrían como plan ideal, su culminación en el medio natural. Es decir, la primera aproximación se hace en el aula o en el centro, para concluir con una salida donde los alumnos puedan practicar lo aprendido en el medio ideal de la actividad.

Dentro de las modernas orientaciones de la Educación Física escolar, los docentes inventan cada vez nuevos modelos y maneras para adaptar y llevar al completo las Actividades y Deportes practicados a diario en el medio natural, a las características específicas del trabajo diario de clase en educación física. El carácter cambiante de la naturaleza va a convertirse en el principal exponente de su desarrollo y precisamente va a ser la incertidumbre asociada a sus imprevisibles 
cambios el principal rasgo que las define y las diferencia.

En un periodo en el que se hace más profunda la alteración estructural de las capacidades y destrezas motoras y cognitivas, deben utilizarse de forma adecuada todas las potencialidades educativas y formativas de la actividad física, ya que sólo de ese modo es posible un desarrollo íntegro y óptimo de nuestros alumnos. Tradicionalmente, las Actividades Física en el Medio Natural se sitúan fuera del espacio curricular debido entre otras cosas, a sus características y al medio donde deberían ser desarrolladas. Por lo que requieren un tratamiento como actividades extraescolares o complementarias, a la vez que deberemos suplir las complicadas barreras burocráticas y organizativas. Y todo ello, para conseguir una educación más equilibrada y completa en los discentes (Baena, 2006).

\section{3.- OBJETIVOS.}

Con el presente trabajo, los objetivos que se han pretendido alcanzar son los siguientes:

- Crear un material curricular fijo para el aula de Educación Física.

- Acercar uno de los contenidos del bloque de Actividades Física en el Medio Natural (la escalada) a los alumnos de Educación Secundaria Obligatoria.

- Iniciar y practicar la escalada deportiva, conociendo sus materiales y contenidos de trabajo.

- Desarrollar en más profundidad el currículum en relación al bloque de contenidos de Actividades en el Medio Natural.

\section{4.- METODOLOGÍA.}

El procedimiento metodológico para llevar a cabo la construcción de esta instalación, ha seguido se divide en varios pasos:

1. Seleccionar el lugar idóneo para la construcción del rocódromo. Para ello, se ha procedido a revisar las paredes del centro con más de $20 \mathrm{~m}^{2}$ para poder construir la instalación. Tras revisar los posibles lugares, se optó por hacerlo dentro del gimnasio por varios motivos:

- Se encuentra en un lugar cerrado, por lo que no le afectaría las inclemencias meteorológicas y así se ahorraría en mantenimiento.
- Por tanto, permite su uso en cualquier época del año.

- El control de su uso es más directo, evitando así ser utilizado por alumnos en horarios no lectivos o por alumnos que no son del centro.

- El control sobre el grupo es mayor, evitando así su dispersión y las posibilidades de riesgo.

- Su mantenimiento es más fácil, puesto que al estar en un lugar donde se trabaja a diario, se puede ir observando periódicamente su estado.

2. Estudiar los materiales para su construcción. Debido a que disponíamos de una pared con espalderas que no se utilizaba normalmente, primeramente optamos por retirar las espalderas y utilizar dicha pared. El problema venía por los materiales de construcción, ya que el tabique está construido con ladrillo y escayola. Los elementos de anclaje que se suelen utilizar en escalda son para colocarlos en rocas macizas, como los spit, los parabolt o los anclajes químicos. Trabajar sobre ladrillo no proporciona ninguna seguridad, puesto que el ladrillo no es un material resistente que nos vaya a ofrecer una gran resistencia.

Una vez revisado esto, se optó por colocar tablones de madera tipo DM de 19mm (mínimo debe ser de $18 \mathrm{~mm}$ ), los cuales anclaríamos a la pared. Pero volvíamos a la misma situación, así que optamos por anclarlos a las espalderas, disponiendo de tres cuerpos (dobles) de espalderas.

Para ello, se estudió el caso y se vio la necesidad de anclar mejor la espalderas a la pared ya que originariamente iban sujetas a ella con dos escuadras de color negro por cada travesaño vertical (6 por espaldera).Cada escuadra lleva un taco y tornillo del $\mathrm{n}^{\circ} 10$ de cabeza hexagonal a la pared y dos tornillos de $1,6 \times 40$ a la madera. Por tanto se han colocado otras 45 escuadras de color blanco de 20x25cms distribuidas como sigue: cinco escuadras por cada travesaño vertical de la espaldera, equidistante una de otra (a $30 \mathrm{~cm}$.), con tres tornillos de anclaje a la pared (para tacos del $n^{\circ}$ 7) y dos a la madera de $1,6 \times 40$. De esta manera se obtiene un total de escuadras colocadas (las negras y las blancas) en número de 57 , siendo capaces de soportar una tensión de más de 22 KN [figura 1] 


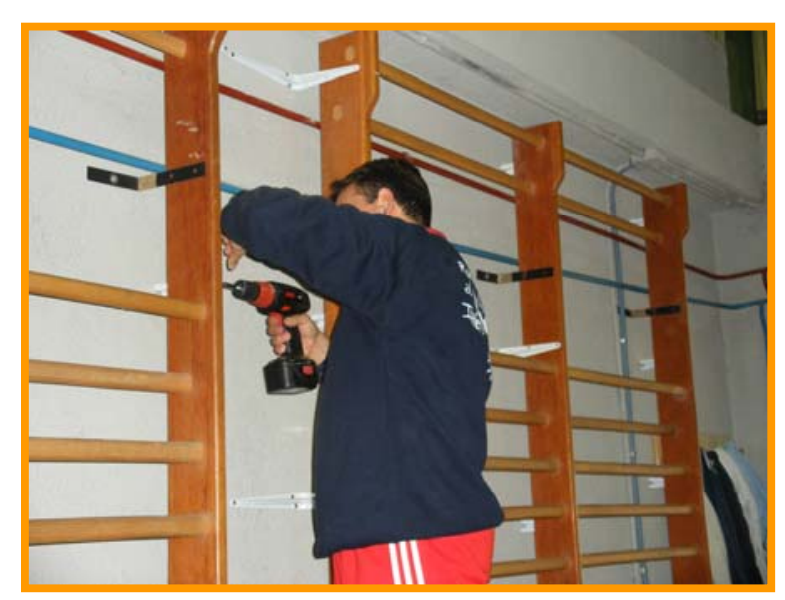

Figura 1.-Fijación de escuadras a espalderas y pared.

Puesto que los tablones de DM se encuentran apoyados sobre el suelo, el peso real recae sobre el mismo, de forma que las espalderas apenan soportan tensión, únicamente cuando los alumnos lo utilizan [figura 2].

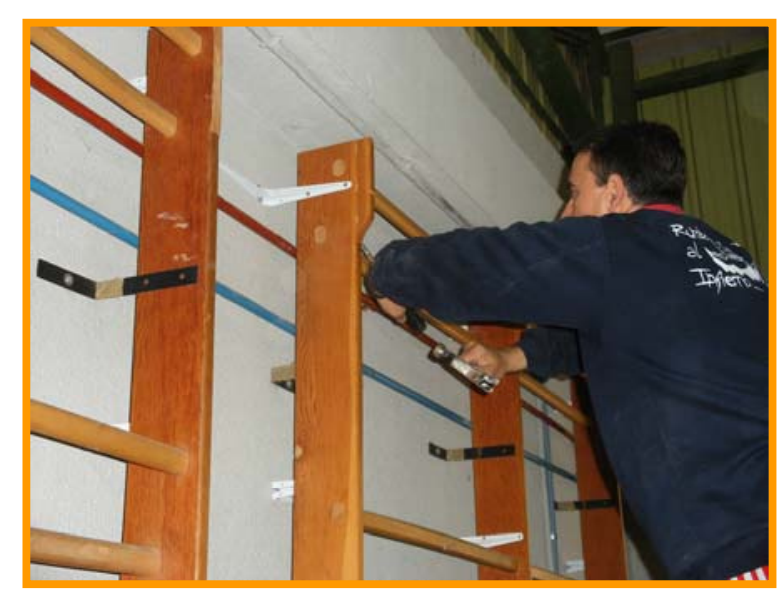

Figura 2.-Colocación de escuadras en la espaldera.

3. Las presas. En el mercado existen gran cantidad de tipos de presas, de plástico, de resina, etc. Puesto que el presupuesto era escaso, se optó por realizar las presas nosotros mismos con maderas sobrantes. Para ello, hay que seguir varios criterios:

- Deben permitir un buen agarre, puesto que estamos en iniciación.

- No deben tener astillas y deben estar pulidas para evitar daño alguno.

- No deben rotar, por lo que la colocación debe ser efectiva.

- Deben ser de diferentes formas y tamaños. Con respecto a la forma, deben permitir utilizar los 5 dedos de la mano, permitir realizar agarres laterales, etc.
Con respecto al tamaño, las podemos clasificar en pequeñas (40-60cms2), medianas $\left(60-80 \mathrm{cms}^{2}\right)$ y grandes (con más de $\left.80 \mathrm{cms}^{2}\right)$.

4. Diseño. Es uno de los trabajos más complejos, puesto que requiere tener cierta experiencia en rocódromos y en escalada. Lo primero que se hizo, fue dividir la superficie de los tablones en metros cuadrados, de manera que se colocarán entre 3-5 presas por m2 para iniciación, 2-3 para avanzados, y 1 para perfeccionamiento. Además, se puede intercambiar con tamaños y formas diferentes que permitan agarren diferentes.

La idea primera es realizar varios recorridos, por colores de presas, donde los alumnos puedan trabajar iniciación, avanzados y perfeccionamiento. De esta forma, se hace el diseño sobre papel y se revisa hasta quedar lo más correcto posible. Una vez trabajando, se transcribe a los tablones señalando los puntos donde irán las presas. Para colocar las presas [figura 3], se utilizan grapas del 12 para rocódromo y tornillos del mismo número. Puesto que la idea es poder diseñar varios circuitos, se colocaron más grapas que presas, con idea de poder ir cambiando las presas de lugar y evitar de esta forma que el rocódromo se haga monótono.

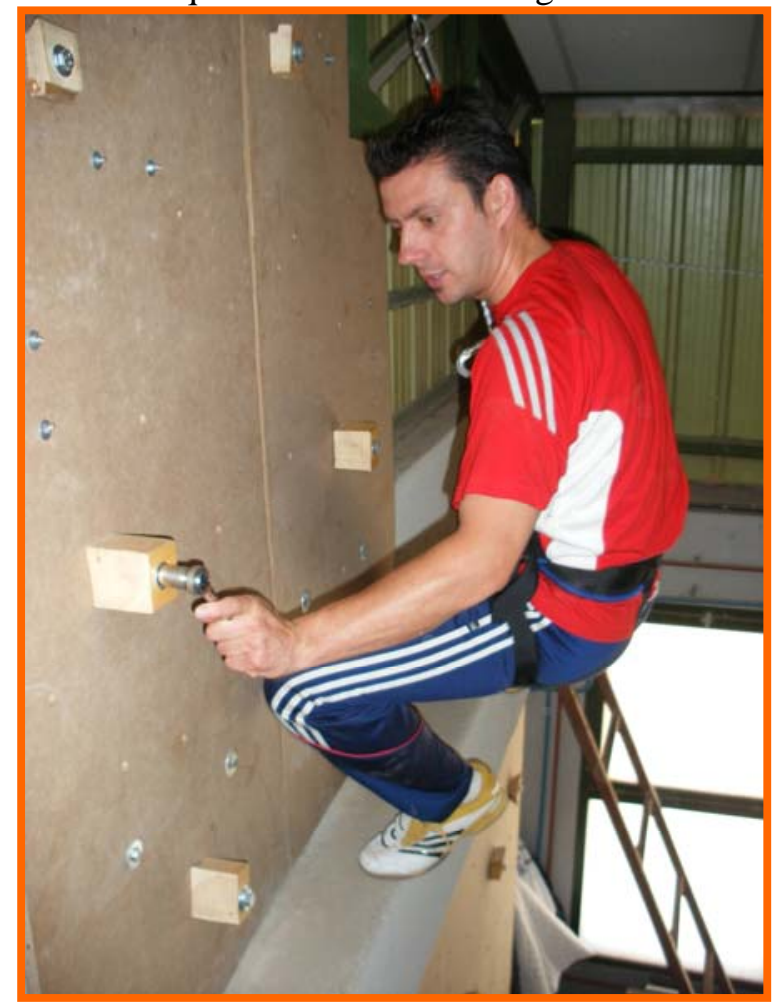

Figura 3.-Colocación de las presas. 
Por último, y una vez diseñado los recorridos y colocadas las presas, se creó dos puntos de reunión (para crear dos vías de escalada) y se colocó varias chapas para trabajar la escalada de primero en cordada.

\section{5.- REPERCUSIÓN SOBRE COMUNIDAD EDUCATIVA.}

LA

La actual conciencia del respeto al medio natural y a la conservación de la naturaleza, es en parte la causante (y a ella damos gracias) de que estas actividades estén presenten en nuestro currículo desde el primer tiempo pedagógico. En este sentido, los diversos decretos de enseñanza identifican objetivos y contenidos relacionados con estas prácticas en la Educación Primaria y Secundaria.

En el sistema educativo actual, las Actividades Físicas en el Medio Natural se abordan desde dos áreas, como son el Área de Conocimiento del medio y el Área de Educación Física (Martínez y Patiño, 2003). Desde la perspectiva de este trabajo, realizaremos un análisis de los elementos del currículo que se ven trabajados a partir de este nuevo material curricular:

Partimos del Primer Nivel de Concreción, propuesto por la Administración Educativa Central que aporta la legislación, hoy en día tenemos que hablar del Decreto 148/2002 de 14 de mayo, por el que se modifica el Decreto 106/1992, de 9 de junio, por el que se establecen las enseñanzas correspondientes a la Educación Secundaria Obligatoria en Andalucía (BOJA n ${ }^{\circ}$ 75 de 27 de junio 2002) y a partir de la LOE (Ley Orgánica 2/2006 de 3 de mayo) y hablamos del Real Decreto 1631/2006, de 29 de diciembre, por el que se establecen las enseñanzas mínimas correspondientes a la Educación Secundaria Obligatoria (BOE 5 de 5 de enero), aún no aplicable en Andalucía. Esta instalación y su uso para la escalada contribuyen a conseguir los siguientes elementos curriculares:

\section{1.- Competencias básicas del área de educación física.}

Antes que los objetivos, en el RD.1631/2006 de 29 de diciembre y en la regulación de las enseñanzas mínimas que se hace en el mismo tiene especial relevancia la incorporación de las competencias básicas que deben alcanzar los alumnos al final de la E.S.O.

En el marco de la propuesta realizada por la Unión Europea, se han identificado ocho competencias, a las que nuestra materia, la Educación física, puede aportar su granito de área. Pero en relación con el contenido que nos atañe en este artículo, contribuiremos de forma más clara y directa con la competencia en el conocimiento y la interacción en el mundo físico, que proporciona conocimientos y destrezas sobre determinados hábitos saludables que acompañarán a los jóvenes más allá de la etapa obligatoria. Además aporta criterios para el mantenimiento y mejora de la condición física. Por otra parte, colabora en un uso responsable del medio natural a través de las actividades físicas realizadas en la naturaleza.

\section{2.- Objetivos generales del área de Educación Física.}

A continuación, vamos a ver la relación con los objetivos generales del área de Educación Física.

a) D. 148/2002 de 14 de mayo, por el que se modifica el Decreto 106/1992, de 9 de junio, por el que se establecen las enseñanzas correspondientes a la Educación Secundaria Obligatoria en Andalucía:

1.- Conocer y valorar su cuerpo, con el fin de contribuir a mejorar sus cualidades físicas básicas y sus posibilidades de coordinación y control motor...

4.- Conocer, diseñar y disfrutar con actividades físicas que, respetando el medio natural, contribuya a su conservación y mejora.

5.- Participar en actividades físicas y deportivas, estableciendo relaciones constructivas y equilibradas con los demás, independientemente del nivel de habilidad alcanzado, y colaborando en la organización de aquellas.

b) RD. 1631/2006 de 29 de diciembre, por el que se establecen las enseñanzas mínimas correspondientes a la Educación Secundaria Obligatoria:

3.- Realizar las tareas dirigidas al incremento de las posibilidades de rendimiento motor, a la mejora de la condición física para la salud y al perfeccionamiento de las funciones de ajuste, dominio y control corporal, adoptando una actitud de autoexigencia en su ejecución.

5.- Planificar actividades que permitan satisfacer las necesidades en relación a las capacidades físicas y habilidades específicas a partir de la valoración del nivel inicial. 
6.- Realizar actividades físico-deportivas en el medio natural que tengan bajo impacto ambiental, contribuyendo a su conservación.

7.- Conocer y realizar actividades deportivas $\mathrm{y}$ recreativas, individuales $\mathrm{y}$ colectivas,...con progresiva autonomía en su ejecución.

8.- Mostrar habilidades y actitudes sociales de respeto, trabajo en equipo,...

\section{3.- Contenidos generales del área de} Educación Física.

Los bloques de contenidos a tener en cuenta son los siguientes:

a) D. 148/2002 de 14 de mayo, por el que se modifica el Decreto 106/1992, de 9 de junio, por el que se establecen las enseñanzas correspondientes a la Educación Secundaria Obligatoria en Andalucía:

\section{5.- Actividad Física en el Medio Natural.}

b) RD. 1631/2006 de 29 de diciembre, por el que se establecen las enseñanzas mínimas correspondientes a la Educación Secundaria Obligatoria:

\section{Actividades en el Medio Natural.}

\section{4.- Relación con los contenidos transversales.}

Siguiendo la Orden de 19 de diciembre de 1995 por la que se establece el desarrollo de la Educación en Valores en Andalucía, las diferentes áreas del currículo deben formar al alumno respecto a una serie de valores que tienen especial relevancia en la sociedad. Consideramos que los temas transversales, por su propio sentido y concepción, no deben entenderse como otras áreas añadidas o como aspectos concretos de determinadas áreas, sino como algo que está impregnando de forma permanente y global el currículo de todas las áreas y materias, y que debe abordarse de una forma sistemática y continuada, como una trabajo conjunto y compartido por todo el equipo docente de esta etapa.

El desarrollo integral del alumno, fin último de Educación Secundaria, no se podrá completar sin la formación en valores que está asociada al tratamiento de los valores y sin la preparación para una adecuada socialización que constituye la esencia de los temas transversales.

Teniendo en cuenta el contenido que estamos tratando, hemos decidido dar prioridad a los siguientes temas transversales:

\section{- Educación para la salud.}

Es evidente que el peso de la Educación para la Salud cae de lleno en el propio currículo del área de Educación Física. En este aspecto incide fundamentalmente el intento de conseguir en el alumnado actitudes positivas frente a varias cuestiones: la higiene corporal en la práctica de actividades físicas, la toma de conciencia de la propia condición física, la valoración del efecto de uno buenos hábitos sobre la condición física y la salud en general, el respeto de las normas de prevención de accidentes en la práctica de la actividad física, la relación de Actividades Físicas en el Medio Natural y la salud,...

\section{- Educación ambiental.}

La Educación Ambiental tendrá que empezar con el medio más cercano a los alumnos (las instalaciones deportivas del centro y el material que se utiliza) como referencia más próxima, conocida y en la que los alumnos inciden con sus comportamientos, para, paulatinamente, conocer y entender las problemáticas medio ambientales más alejadas y globales.

La Educación Física es un área que contribuye de forma importante a la Educación Ambiental del alumnado. Desde el punto de vista conceptual, puesto que la actividad física es un instrumento de exploración a través del cual el alumno conoce e interactúa con lo que le rodea, y servirá para que identifique los elementos que componen ese entorno y la utilidad de cada uno de ellos.

En lo procedimental se han de incorporar todos los contenidos relativos al uso adecuado del entorno natural, empezando por los relacionados con la observación para reconocer cada entorno, sus posibilidades para realizar actividades físicas, y la detección de los cuidados a tener en cuenta para no deteriorarlo.

$\mathrm{Y}$, por supuesto, incorporaremos el desarrollo de actitudes de cuidado del entorno y de valores de responsabilidad y cooperación para su defensa y conservación. Todo ello, aprovechado el marco incomparable donde habitan los alumnos/as, el cual será escenario de muchas actividades propuestas por el centro para todos los alumnos en general.

- Educación para la igualdad de oportunidades para ambos sexos.

Todos los contenidos constituyen una fuente continua de oportunidades para resaltar la igualdad entre sexos. Para ello será necesario ofrecer distintas alternativas como la escalada para que alumnos $y$ alumnas conozcan $y$ 
practiquen esta actividad y poder adaptarse a los distintos intereses, preferencias y capacidades. Estas prácticas, además presentan las características de ser no sexistas, presentando igualdad de oportunidades para chicas y chicos.

- Educación para la paz.

Los contenidos del área de Educación Física y el de la escalada en particular, coinciden con actividades de integración entre iguales y de interculturalidad. Resaltan la importancia de las reglas y normas y de su respeto. Como fin último, incorporaremos los contenidos actitudinales necesarios para el trabajo cooperativo, tan importante en estas actividades.

\section{5.- Otras posibles relaciones.}

Este trabajo también conlleva su posible relación interdisciplinar con otras áreas, como por ejemplo, contenidos relacionados con:

$\square$ Matemáticas: contenidos relacionados con trayectorias, fuerzas, presiones, tracciones,...

$\square$ Dibujo: líneas rectas, ángulos, geomorfología de las presas,...

$\square$ Inglés: cierto conocimiento de palabras como boulder, cinta express, etc.

$\square$ Ciencias. Naturales: respeto por el medio natural y verlo como un lugar de práctica deportiva, ayuda al conocimiento del cuerpo, al conocimiento de los materiales,...

$\square$ Física: de manera interdisciplinar se habla de fuerza de la gravedad, magnitud de resistencia de los materiales, agarres, ángulos y tracciones, poleas (en escalada top rop), etc.

$\square$ Lenguaje: vocabulario específico como chapas, rocódromo, mosquetón,...

$\square$ Tecnología: material y herramientas para construir el rocódromo,...

\section{6.- EVALUACIÓN.}

Dentro de la Evaluación, se llevará a cabo el siguiente trabajo:

- Por parte del alumno se realizará un cuestionario con preguntas de tipo abiertas y categorizadas (Sierra, 2001). Dentro de estas preguntas se les cuestionará sobre su interés hacia la instalación, sobre sus posibilidades educativas, sobre si se puede trabajar la condición física, si ayuda a mejorar las relaciones con sus compañeros/as, etc.

- Por parte del profesor, cuando se realice la primera U. D. de escala que se llevará a cabo con $4^{\circ}$ de la ESO en el mes de febrero, se realizará una evaluación, a través de una hoja de registros, de los objetivos propuestos en dicha unidad y conseguidos gracias a esta instalación. También se deberían evaluar las condiciones de uso y de seguridad de forma periódica (antes de comenzar cada unidad didáctica relacionada con el contenido de escalada).

- Por último, comentar que está pendiente su revisión y homologación por parte de un agente externo (arquitecto o federación deportiva) para ver su adecuación a las normas europeas de instalaciones de escalada.

\section{5.- REFERENCIAS BIBLIOGRÁFICAS.}

Baena Extremera, A. (2006). Tema 47. Actividades en el medio natural. Tipos, clasificaciones y recursos. Organización de actividades física en la naturaleza. No publicado. Temario de Oposiciones para el cuerpo de Educación Secundaria. Inédito.

Carlson, T. B. (1995). We hate gym: student alienation from Physical Education. Journal of Teaching in Physical Education, 14(4), 467-477.

Carreño, M. (2000). Teorías e instituciones contemporáneas de educación. Madrid: Síntesis.

Cossio, M. B. (1888). Colonias escolares de vacaciones. Madrid: BILE, XII.

Decreto 105/1992 de 9 de junio, que establece las enseñanzas correspondientes a la Educación Primaria en Andalucía. En Boletín Oficial de la Junta de Andalucía, núm. 56 de 20 de junio de 1992.

Decreto 148/2002 de 14 de mayo, por el que se modifica el Decreto 106/1992, de 9 de junio, por el que se establecen las enseñanzas correspondientes a la Educación Secundaria Obligatoria en Andalucía. En Boletín Oficial de la Junta de Andalucía, número 75 de 27 de junio 2002.

Decreto 208/2002 de 3 de Julio por el que se modifica el Decreto 126/1994 por el que se establecen las enseñanzas correspondientes a 
Bachillerato en Andalucía. En Boletín Oficial de la Junta de Andalucía, número 97 de 20 de agosto de 2002.

Devís Devís, J. (1994). Educación Física y desarrollo del currículo. Un estudio de casos en investigación colaborativa. Tesis doctoral, Universidad de Valencia, Valencia.

Dewar, A. (1991). Feminist pedagogy in physical education: promises, possibilities and pitfalls. Journal of Teaching in Physical Education, 62(6), 68-77.

Fernández-Rio, J., Medina Gómez, J. F. \& Garro García, J. (2003). La transformación del aula de Educación Física en un espacio de acción y aventura a través de los desafíos físicos cooperativos. En Fuentes García P. \& Bellido Pérez, M. (coord.), Primer Congreso Europeo de Educación Física FIEP (pp. 347-353). Cáceres: FIEP.

García Fernández, P. \& Quintana Valverde, M. (2005). Introducción a las Actividades en la Naturaleza. Sevilla: Wanceulen.

Ley Orgánica de Educación del 2/2006 de 3 de Mayo (BOE, 4-5-2006).

Lagardera, F. (2000). Perspectivas de una Educación Física integral para el siglo XXI. Tándem (1), 67-78.

López Serra, F. (1998). Historia de la Educación Física. La institución libre de enseñanza. Madrid: Gymnos.
Martínez Lareo E. J. \& Patiño Calviño, D. (2003). Propuesta para el desarrollo de actividades en la naturaleza en el entorno de un centro educativo. Revista de Educación Física (90), 29-35.

Orden de 19 de diciembre de 1995 por la que se establece el desarrollo de la Educación en Valores en Andalucía. En Boletín Oficial de la Junta de Andalucía, número 9 de 12 de enero de 1996.

Pastor Pradillo, L. (1997). Historia de la Educción Física en la España. El ámbito profesional (1883-1961). Tesis doctoral, Universidad de Valladolid, Valladolid.

Povenius, M. \& Romar, J. E. (1995). Is the low skilled student lost in P.E.?. A.E.I.S.E.P. World Congress, Israel. Wintage Institute of Physical Education.

Real Decreto 1631/2006 de 29 de Diciembre por el que se establecen las enseñanzas mínimas correspondientes a la Educación Secundaria Obligatoria. En Boletín Oficial del Estado, número 5 de 5 de enero de 2007.

Sage, G. H. (1998). Power and ideology in American Sport. Champaing, IL: Human Kinetics.

Sierra Bravo, R. (2001). Técnicas de investigación social. Teoría y ejercicios. Madrid: Paraninfo Thomson Learning. 